\title{
Mesure ambulatoire de la force maximale volontaire isométrique du quadriceps chez le patient BPCO
}

\author{
Maximal isometric voluntary quadriceps strength assessment in \\ COPD
}

\section{MOTS CLÉS BPCO ; Muscle quadriceps ; Force musculaire ; Diagnostic ; Faiblesse musculaire}

\section{KEYWORDS COPD;} Quadriceps muscle; Muscle strength; Diagnosis; Muscle weakness

\author{
D. Bachasson ${ }^{a}$, E. Villiot-Danger ${ }^{b}$, S. Verges ${ }^{a}$, \\ M. Hayot ${ }^{c}$, T. Perez ${ }^{d}$, A. Chambellan ${ }^{\mathrm{e}}$, B. Wuyam ${ }^{\mathrm{a}, *}$
}

a Inserm U1042, laboratoire HP2, pôle locomotion, rééducation et physiologie, clinique physiologie, sommeil et exercice, $\mathrm{CHU}$ de Grenoble, université de Grenoble, 38000 Grenoble, France

${ }^{\mathrm{b}}$ Centre de pneumo-allergologie, les acacias, 05107 Briançon, France

c Inserm U1046, département de physiologie, service physiologie clinique, hôpital Arnaud de Villeneuve, $\mathrm{CHU}$ de Montpellier, 34295 Montpellier, France

d Service d'explorations fonctionnelles respiratoires et clinique des maladies respiratoires, hôpital Calmette, CHRU de Lille, 59037 Lille, France

e Inserm U1087, institut du thorax, CHU de Nantes, 44093 Nantes, France

\section{Contexte et objectifs}

Le retentissement extra-pulmonaire de la BPCO sur la fonction neuromusculaire périphérique reçoit une attention particulière du fait de son influence directe sur la tolérance à l'exercice [1]. Ainsi, la diminution de l'activité physique spontanée mais également des facteurs systémiques (stress oxydant, inflammation, hypoxie, diminution des hormones anaboliques) contribuent de façon majeure à la détérioration de la fonction musculaire périphérique des patients porteurs d'une BPCO [2]. Ces altérations se traduisent principalement par une amyotrophie (i.e. diminution du nombre et du diamètre des myofibrilles) entraînant une faiblesse musculaire [3-5] et par une réduction des capacités oxydatives [6] engendrant une augmentation de la fatigabilité (i.e. réduction des capacités contractiles induites par l'exercice) [7]. La faiblesse des muscles des membres inférieurs, et particulièrement du quadriceps apparaît comme fortement liée à la morbidité et la mortalité des patients [8]. Ainsi, la sévérité de l'amyotrophie du quadriceps a été montrée comme étant un meilleur prédicteur de la mortalité que l'indice de masse corporelle [9] et est associée à une plus grande consommation de soins de santé [10]. Avec le même degré

\footnotetext{
* Auteur correspondant. Inserm U1042, laboratoire HP2, UF recherche sur l'exercice, hôpital Sud, avenue Kimberley, 38434 Échirolles, France.

Adresse e-mail : bwuyam@chu-grenoble.fr (B. Wuyam).
} 
d'obstruction bronchique, un patient présentant une faiblesse du quadriceps a 13 fois plus de risque de décéder dans les 5 ans qu'un patient avec une force musculaire préservée [9]. Cette faiblesse peut être présente à des stades très précoces de l'évolution de la maladie $(\sim 30 \%$ des patients GOLD I/II [11]) et chez des patients avec des indices de masse corporel normaux [12].

La diffusion de l'évaluation de la force du quadriceps dans le cadre clinique apparaît donc comme un enjeu majeur pour caractériser les patients, orienter les interventions thérapeutiques, évaluer leur efficacité et mesurer le retentissement d'un épisode clinique telle qu'une exacerbation $[13,14]$. Les principales indications et contre-indications de la mesure sont résumées dans le Tableau 1. Une technique idéale devrait pouvoir être utilisée aussi bien chez le patient ambulatoire (consultation de pneumologie/réentraînement à l'effort) ou hospitalisé [15]. Dans ce cadre, l'évaluation de la force maximale volontaire (FMV) isométrique par dynamométrie manuelle s'est révélée être un moyen d'évaluation simple, comparable aux techniques de références $[16,17]$ et reproductible [18]. Cette méthode est fréquemment décrite dans la littérature comme hand-held dynamometry (HHD) $[15,19]$. Nous verrons que l'utilisation proposée par les groupes de travail «Groupe Fonction» et «GTK» de la SPLF n'est pas hand-held (tenue à la main) mais utilise une sangle inextensible afin de limiter les biais lors de la mesure. Cette technique est largement inspirée des travaux de Bohannon et al. [20] qui décrivent une technique par dynamométrie manuelle «stabilisée». Cependant, il n'existe pas à ce jour de description détaillée et standardisée de la méthode ce qui limite sa diffusion dans le cadre clinique. Nous proposons donc, dans cette fiche technique à l'attention des pneumologues, des kinésithérapeutes et tout professionnel de santé impliqué dans la prise en charge de patients BPCO, une méthode standardisée pour faciliter la mise en place de cette évaluation.

\section{Dynamomètre}

Plusieurs choix de dynamomètres peuvent être envisagés. Les critères devant être pris en compte pour la sélection d'un appareil sont: sa portabilité, sa gamme de mesure $(0-1000 N)$, sa résolution $(\sim 1 N)$, mémoire de mesure maximale, la stabilité de sa mesure dans le temps (garantie par le constructeur (étalonnage périodique) ou possibilités de calibration manuelle), son adaptabilité à la chaîne de mesure proposée dans cette fiche technique, éventuellement un marquage CE médical (acquisition de l'appareil par des structures de soins) et une interface informatique (biofeedback et/ou acquisition de données). Dans le cadre de cette fiche technique, nous avons choisi à titre d'exemple le MicroFet2 ${ }^{T M}$ (Hoggan Health Industries, États-Unis) qui réunit tous les critères précédemment cités. Ce type d'appareil a fait ses preuves dans de nombreuses études cliniques [21] comprenant notamment la mesure de la force maximale isométrique du quadriceps [20]. En fait, tout autre appareil remplissant les critères ci-dessus et permettant de respecter la position de mesure décrite ci-après est acceptable qu'il s'agisse de dynamomètres manuels (ex. MicroFet2 ${ }^{T M}$; Lafayette Manual Muscle Testing, Lafayette Instrument, États-Unis) ou non manuels (ex. Dynamax, Electronic Conseil, France).

\section{Matériel complémentaire}

Un plan de travail suffisamment haut est nécessaire pour permettre au patient de s'asseoir sans que ses pieds ne touchent le sol (Fig. 1). Ce plan doit être le plus dur possible, il peut s'agir d'un bureau ou d'une table d'examen avec une mousse dense. Il faut cependant éviter les surfaces trop lisses et glissantes. Il doit idéalement disposer d'un pied solide légèrement en retrait par rapport au bord du plan. Un lit n'est pas adéquat. Un stylo dermographique et un mètre ruban sont également nécessaires. Une sangle inextensible réglable du même type que celles utilisées pour le chargement (ex. galerie automobile, largeur comprise entre 2 et $6 \mathrm{~cm}$ ) est nécessaire pour installer la chaîne de mesure décrite ci-dessous. Un coussin en coin d'environ $5 \mathrm{~cm}$ de hauteur et d'une forte densité $\left(>140 \mathrm{~kg} \cdot \mathrm{m}^{-3}\right)$ permettra d'optimiser la position du patient. Des pièces de mousse dense sont également nécessaires pour optimiser le confort du patient et faciliter l'enveloppement du dynamomètre (voir ci-dessous). Un matériel sensiblement différent pourra être nécessaire en fonction du type de dynamomètre utilisé et du montage souhaité.

\section{Installation de la chaîne de mesure}

Plusieurs montages sont envisageables, dépendant en partie du type de dynamomètre utilisé (Fig. 2). Dans le cas particulier du MicroFet2 ${ }^{\mathrm{TM}}$ (et plus généralement celui des dynamomètres manuels), la sangle est fixée au pied de la table d'examen/bureau via un nœud de cabestan

\begin{tabular}{ll}
\hline Tableau 1 & Indications et contre-indications de la mesure de la force maximale isométrique du quadriceps chez le BPCO. \\
\hline Indications & Contre-indications \\
\hline Diagnostic & Absolues \\
Évaluation du retentissement d'une exacerbation & Antécédents interdisant la réalisation d'effort maximaux à \\
Effet d'un traitement pharmacologique ou non & glotte fermée (ex. cardiomyopathie non stabilisée, hernie \\
pharmacologique & abdominale ou inguinale, etc.) \\
Bilan pré-réentrainement à l'effort & Relatives \\
Aide à l'individualisation de la prise en charge en & Antécédents limitant la réalisation d'une extension \\
cohérence avec le tableau clinique et les symptômes & maximale du genou (arthrose, arthrite, traumatismes...) \\
\hline
\end{tabular}




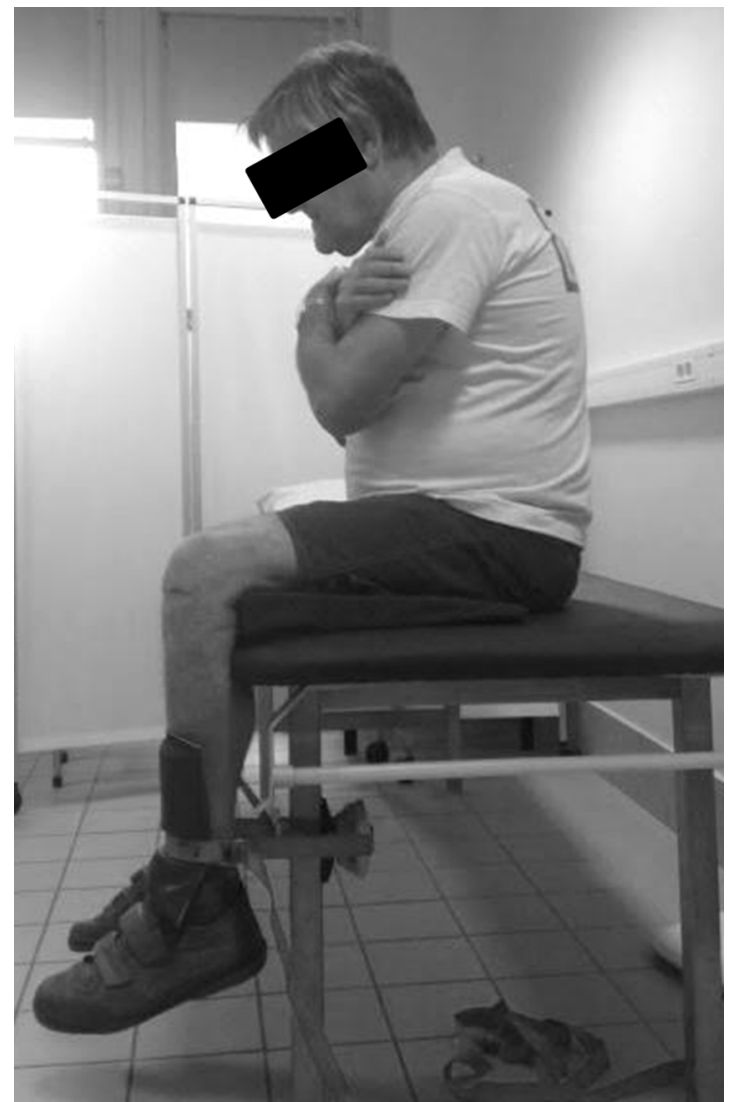

Figure 1. Vue d'ensemble du dispositif et d'un patient réalisant une contraction maximale volontaire du quadriceps.

permettant le réglage de la hauteur de la sangle. Le dynamomètre est ensuite interposé entre la sangle et le tibia du patient. Une pièce de mousse est intercalée entre le capteur et le tibia et une autre pièce de mousse est intercalée entre la sangle et le dynamomètre. Dans ce cas, l'opérateur stabilisera le dispositif en veillant à ne pas appliquer de force supplémentaire (Fig. 2A). Une alternative est de fixer le dynamomètre à l'arrière (dans le cas d'un dynamomètre fonctionnant en compression comme le MicroFet2 ${ }^{\mathrm{TM}}$ ) ou à l'avant (dans le cas d'un dynamomètre fonctionnant en traction) du pied de la table d'examen/bureau. L'embout du capteur utilisé est celui qui s'adapte le mieux au pied de la table ou bureau. Dans le cas du MicroFet2 ${ }^{T M}$, la sangle réglable prend appui sur la face postérieure du capteur et contourne la face antérieure de la jambe du patient (Fig. 2B).

\section{Préparation du sujet}

Le centre du point d'appui du dynamomètre ou de la sangle est repéré en traçant un trait horizontal au stylo dermographique $5 \mathrm{~cm}$ au-dessus de la malléole médiale du patient (Fig. 3). Le centre articulaire du genou (i.e. assimilé à l'interligne fémoro-tibial) est repéré par un trait horizontal au niveau des plateaux tibiaux. L'opérateur mesure ensuite la distance entre les deux traits qui permettra la mesure du moment de la force produite par le patient (Fig. 4).

\section{Installation du sujet}

Le patient ne dispose pas d'appui dorsal et une légère cyphose est demandée afin de limiter la modification de l'angle articulaire de la hanche (Fig. 1 et 4). En effet, toute modification de cet angle entraîne un allongement du droit fémoral qui peut engendrer une majoration de la force musculaire produite (relation tension-longueur). Les membres supérieurs sont croisés sur la poitrine pendant toute la durée de la mesure. La mesure doit s'effectuer avec le genou et la hanche fléchis à $90^{\circ}$. Afin que ces angulations soient maintenues pendant la réalisation d'une contraction maximale volontaire (CMV), il est nécessaire d'utiliser le coussin en coin afin de garantir l'horizontalité du segment fémoral et de fournir un contre-appui poplité. La densité importante de ce dernier permet de limiter son écrasement pendant la CMV qui engendrerait une perte de charge et une modification de l'angle de flexion genou. Classiquement, les mesures sont réalisées unilatéralement et parfois bilatéralement. Dans le cas d'une gonalgie, la mesure est réalisée sur le genou le moins douloureux. Il est ensuite nécessaire de demander au sujet de réaliser une extension de genou afin d'ajuster la longueur de la sangle jusqu'à d'obtenir une flexion de genou à $90^{\circ}$ et de s'assurer de l'horizontalité de la sangle en modifiant sa hauteur. Il est également important de s'assurer que la jambe du patient est parfaitement alignée avec le pied de la table d'examen/bureau.

\section{Réalisation de la mesure}

La mise en place de la chaîne de mesure permet quelques contractions qui constituent à elles seules un léger échauffement. La consigne donnée au sujet est la suivante:

«Le but de ce test est de mesurer votre force maximale. Le mouvement demandé est une extension de la jambe par rapport à la cuisse. Vous devez fournir la plus grande force possible, c'est très important. Je vous indiquerai par un décompte le moment ou vous devrez pousser puis je vous encouragerai pendant plusieurs secondes. Ensuite seulement vous pourrez relâcher. Pendant le test, je vous demande de garder les bras croisés sur la poitrine, de faire le dos rond sans vous pencher en avant et de ne pas vous redressez. Nous répèterons plusieurs fois cet exercice. II s'agit d'un test maximal ». Les encouragements doivent être formulés très vigoureusement avec la même intensité pendant tous les essais: «Allez, allez, allez, allez, etc.». Le temps de contraction est de $4 \mathrm{~s}$ minimum et $6 \mathrm{~s}$ maximum avec un temps de récupération de $30-60 \mathrm{~s}$ entre chaque essai. Les deux premières mesures sont exclues (poursuite de l'échauffement, évaluation de la stabilité de la chaîne de mesure, familiarisation du patient) puis cinq mesures sont la plupart du temps suffisantes pour obtenir trois valeurs variant de moins de $5-10 \%$. La valeur maximale parmi les trois valeurs les plus reproductibles est retenue. On calcule ensuite le moment de la force produite (Fig. 4). 

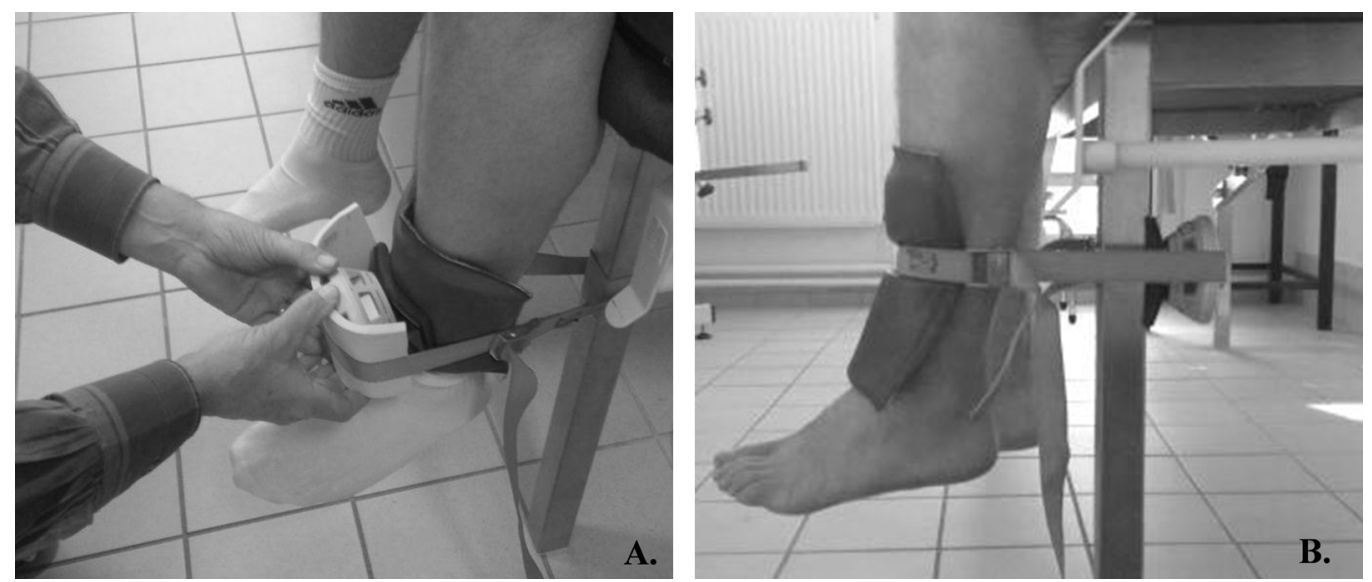

Figure 2. Installations possibles de la chaîne de mesure dans le cas d'une utilisation du MicroFet2 ${ }^{\top M}$ intercalé entre la sangle inextensible et la jambe du patient avec stabilisation passive par l'opérateur (A) ou fixé sur le pied de la table d'examen (B).
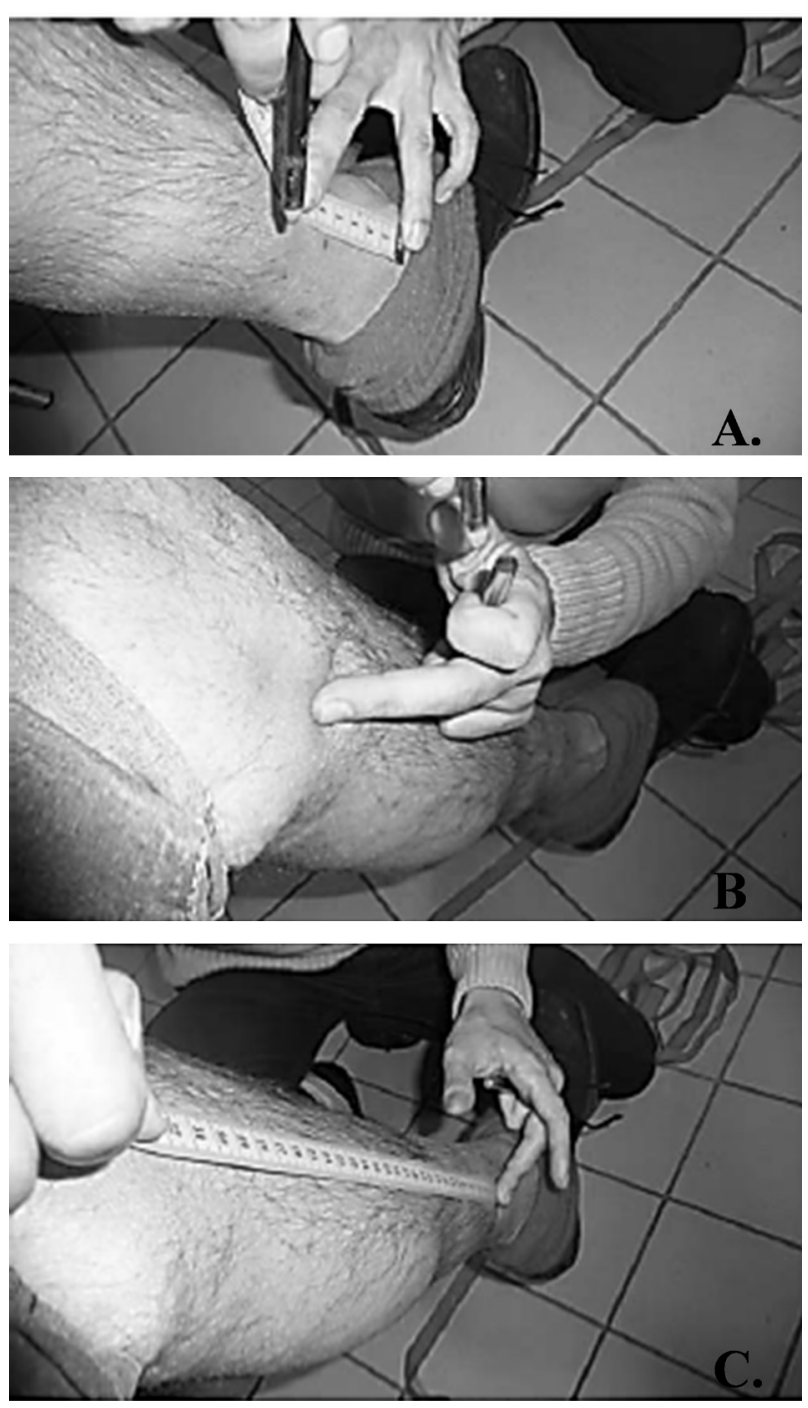

Figure 3. Préparation du patient. Repérage de la hauteur d'appui de la sangle, $5 \mathrm{~cm}$ au-dessus de la malléole médiale $(A)$; repérage de l'interligne articulaire du genou (B); mesure de la distance entre les deux traits correspondant au bras de levier (C).

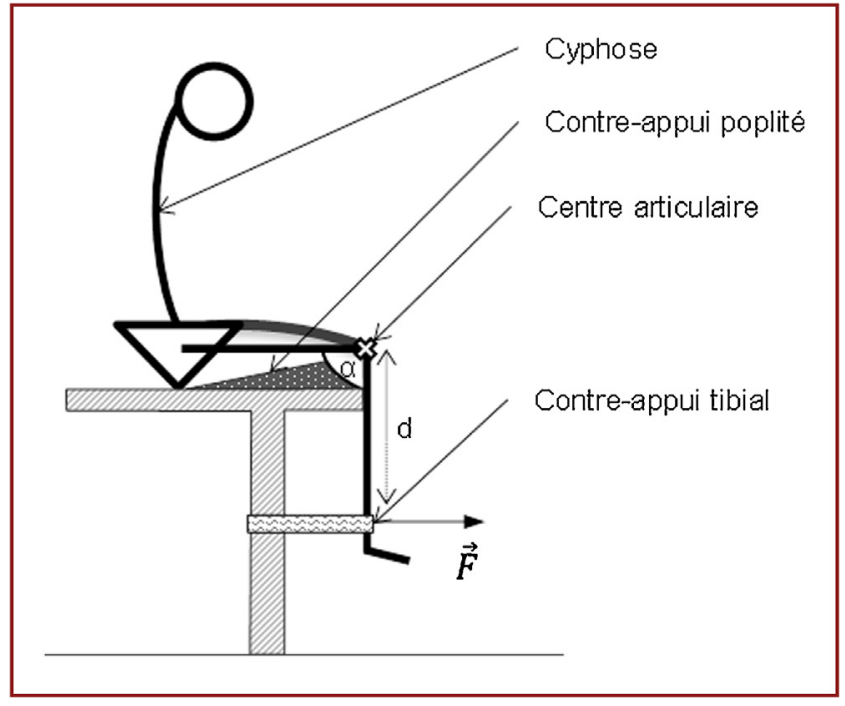

Figure 4. Représentation schématique des conditions de réalisation de la mesure de la force maximale volontaire isométrique du quadriceps et calcul du moment de force produit. Lors de la réalisation contraction maximale volontaire du quadriceps, le patient génère une force $F$ appliquée sur le contre-appui tibial (sangle ou dynamomètre). Le moment de force $(\mathrm{Nm})$ produit par le patient vaut $F(\mathrm{~N}) \times \mathrm{d}(\mathrm{m}) \times \sin \alpha$ soit $F(\mathrm{~N}) \times \mathrm{d}(\mathrm{m})$ avec $\sin 90^{\circ}=1 . \alpha$ : angle de flexion du genou $\left(90^{\circ}\right) ; \mathrm{d}$ : distance du centre articulaire du genou au point d'application de la force.

\section{Valeurs normales}

Nous proposons d'utiliser les équations prédictives de FMV du quadriceps en $\mathrm{Nm}$ conçues par Hogrel et al. [22] du fait de la forte similarité des conditions de réalisation. Pour la jambe droite, $\mathrm{FMV}(\mathrm{Nm})=66,37-(0,87$ $\times$ âge $)+(46,09 \times$ Sexe [0 si femme, 1 si homme $)+(1,21$ $\times$ poids $[\mathrm{kg}])$. Pour la jambe gauche, $\operatorname{FMV}(\mathrm{Nm})=78,00$ $-(0,87 \times$ âge $)+(49,70 \times$ sexe $[0 \mathrm{si}$ femme, 1 si homme] $)$ $+(0,96 \times$ poids $[\mathrm{kg}])$. Il est également possible d'utiliser d'autres valeurs normales telles que celle de Seymour et al. [11] qui prennent en compte la masse grasse corporelle mais qui fournissent une valeur prédictive en $\mathrm{kg}$ et non en $\mathrm{Nm}$. 
Tableau 2 Principaux éléments à retenir pour la mesure de la force maximale isométrique du quadriceps chez le patient BPCO.

À retenir :

Capteur adapté, chaîne de mesure stable, confort du patient

Plan dur, contre-appui poplité, pas de dossier, patient bras croisés et légère cyphose

Explication claire et rigoureuse de l'objectif de la mesure et de la procédure au patient, encouragements vigoureux et systématiques

Observation du patient, répétition-reformulation des consignes

\section{Causes d'erreurs fréquentes}

Outre la motivation des sujets, certaines conditions de réalisation peuvent engendrer une grande variabilité de la mesure voir des valeurs aberrantes. Les causes d'erreurs les plus fréquentes sont les suivantes:

- s'assurer que la chaîne de mesure est bien stable, que la sangle reste bien horizontale et que la jambe du patient est bien alignée avec le pied de la table d'examen/bureau;

- réexpliquer au patient que c'est un effort maximal qui lui est demandé ;

- vérifier et corriger les compensations les plus courantes: rotation du tronc, rotation latérale de la hanche homolatérale, extension du genou hétérolatéral, extension du rachis, redressement, soulèvement des fesses;

- il est demandé au patient de maintenir une légère cyphose. Cependant, cette dernière ne doit pas être accompagnée d'une flexion du tronc qui a pour conséquence déplacement en avant du centre de gravité facilitant le redressement. Le patient a ainsi tendance à se relever pensant la mesure. Expliquer au patient qu'il ne doit pas se pencher en avant.

Les principaux éléments à retenir pour la mesure de la force maximale isométrique du quadriceps chez le BPCO sont présentés dans le Tableau 2.

\section{Conclusion}

La méthode proposée dans cette fiche technique devrait permettre de favoriser la réalisation de l'évaluation du retentissement de la BPCO sur la fonction musculaire périphérique. La mise en pratique de cette évaluation pourrait également favoriser l'élargissement des indications vers d'autres pathologies chroniques à retentissement musculaire périphérique à point de départ pulmonaire ou non pulmonaire.

\section{Déclaration d'intérêts}

Les auteurs déclarent ne pas avoir de conflits d'intérêts en relation avec cet article.

\section{Remerciements}

Le présent travail a été élaboré à partir d'un workshop organisé par les groupes de travail «Groupe Fonction » et «GTK» de la SPLF. Les auteurs sont reconnaissants aux participants de ce workshop: Philippe Joud, Marc Beaumont, Jacques Saugier, Caroline Dubois, Laurence Pittet-Barbier, Pascale Surpas, Jean-Marie Grosbois, Benoît Wallaert, Frédérique Costes, Vincent Tiffreau. Nous remercions également tous les volontaires et patients qui ont permis la réalisation de cette fiche technique. Nous remercions les laboratoires Boehringer-Ingelheim et Genzyme pour leur soutien pour la tenue du premier workshop du «Groupe Fonction» sur le cette thématique. Cette aide logistique ne constitue pas un lien d'intérêt direct ou indirect avec le contenu du présent article.

\section{Références}

[1] Gosselink R, Troosters T, Decramer M. Peripheral muscle weak ness contributes to exercise limitation in COPD. Am J Respir Crit Care Med 1996;153:976-80.

[2] Caron MA, Debigare R, Dekhuijzen PN, et al. Comparative assessment of the quadriceps and the diaphragm in patients with COPD. J Appl Physiol 2009;107:952-61.

[3] Bernard S, LeBlanc P, Whittom F, et al. Peripheral muscle weakness in patients with chronic obstructive pulmonary disease. Am J Respir Crit Care Med 1998;158:629-34.

[4] Pitta F, Troosters T, Spruit MA, et al. Characteristics of physical activities in daily life in chronic obstructive pulmonary disease Am J Respir Crit Care Med 2005;171:972-7.

[5] Polkey MI, Moxham J. Attacking the disease spiral in chronic obstructive pulmonary disease: an update. Clin Med 2011;11:461-4.

[6] Whittom F, Jobin J, Simard PM, et al. Histochemical and morphological characteristics of the vastus lateralis muscle in patients with chronic obstructive pulmonary disease. Med Sci Sports Exerc 1998;30:1467-74.

[7] Swallow EB, Gosker HR, Ward KA, et al. A novel technique for nonvolitional assessment of quadriceps muscle endurance in humans. J Appl Physiol 2007;103:739-46.

[8] Swallow EB, Reyes D, Hopkinson NS, et al. Quadriceps strength predicts mortality in patients with moderate to severe chronic obstructive pulmonary disease. Thorax 2007;62:115-20.

[9] Marquis K, Debigare R, Lacasse Y, et al. Midthigh muscle cross sectional area is a better predictor of mortality than body mass index in patients with chronic obstructive pulmonary disease. Am J Respir Crit Care Med 2002;166:809-13.

[10] Decramer M, Gosselink R, Troosters T, et al. Muscle weakness is related to utilization of health care resources in COPD patients. Eur Respir J 1997;10:417-23.

[11] Seymour JM, Spruit MA, Hopkinson NS, et al. The prevalence of quadriceps weakness in COPD and the relationship with disease severity. Eur Respir J 2010;36:81-8.

[12] Schols AM, Soeters PB, Dingemans AM, et al. Prevalence and characteristics of nutritional depletion in patients with stable COPD eligible for pulmonary rehabilitation. Am Rev Respir Dis 1993;147:1151-6.

[13] Mador MJ, Kufel TJ, Pineda LA, et al. Effect of pulmonary rehabilitation on quadriceps fatiguability during exercise. Am J Respir Crit Care Med 2001;163:930-5.

[14] O’Donnell DE, McGuire M, Samis L, et al. General exercise training improves ventilatory and peripheral muscle strength and endurance in chronic airflow limitation. Am J Respir Crit Care Med 1998;157:1489-97. 
[15] Saey D, Troosters T. Measuring skeletal muscle strength and endurance, from bench to bedside. Clin Invest Med 2008;31:E307-11.

[16] Bohannon RW, Bubela DJ, Wang YC, et al. Adequacy of beltstabilized testing of knee extension strength. J Strength Cond Res 2011;25:1963-7.

[17] Whiteley R, Jacobsen P, Prior S, et al. Correlation of isokinetic and novel hand-held dynamometry measures of knee flexion and extension strength testing. J Sci Med Sport 2012;15:444-50.

[18] O'Shea SD, Taylor NF, Paratz JD. Measuring muscle strength for people with chronic obstructive pulmonary disease: retest reliability of hand-held dynamometry. Arch Phys Med Rehabil 2007;88:32-6.
[19] Bohannon RW. Hand-held dynamometry: adoption 1900-2005. Percept Mot Skills 2006;103:3-4.

[20] Bohannon RW, Kindig J, Sabo G, et al. Isometric knee extension force measured using a hand-held dynamometer with and without belt-stabilization. Physiother Theory Pract 2012;28:562-8.

[21] Groeneweg G, Huygen FJ, Niehof SP, et al. Effect of tadalafil on blood flow, pain, and function in chronic cold complex regional pain syndrome: a randomized controlled trial. BMC Musculoskelet Disord 2008;9:143.

[22] Hogrel JY, Payan CA, Ollivier G, et al. Development of a French isometric strength normative database for adults using quantitative muscle testing. Arch Phys Med Rehabil 2007;88: 1289-97. 